\title{
Erratum to: Improving Treatment Adherence in Schizophrenia
}

Peggy El-Mallakh, PhD, RN, PMHNP-BC $C^{*}$ Jan Findlay, PhD, RN, PMHNP-BC

\section{Address}

*College of Nursing, University of Kentucky, 315 College of Nursing Building, \#547, Lexington, KY, 40536-0232, USA

Email: peggy.el-mallakh@uky.edu

Published online: 11 June 2016

C Springer International Publishing AG 2016

The online version of the original article can be found at http://dx.doi.org/ 10.1007/s40501-016-0074-9.

\section{Erratum to: Curr Treat Options Psych DOI 10.1007/s40501-016-0074-9}

For Tables 1 and 2, the bulleted interventions should correspond to the beginning sentences noted in the findings sections.

The correct Tables are now presented on the next page. 
Table 1. Patient factors that influence treatment adherence

\begin{tabular}{|c|c|c|}
\hline Treatment type & Facilitators to adherence & Findings \\
\hline Psychiatric medication & $\begin{array}{l}\text { - Awareness of illness, positive expectations } \\
\text { medication } \\
\text { will be effective, strong provider-patient } \\
\text { relationship. } \\
\text { - Desire to live normal life, freedom from psychotic } \\
\text { symptoms. } \\
\text { - People with first-episode illness: desire for relapse } \\
\text { prevention, immediate benefit from medications, } \\
\text { family support, alliance with } \\
\text { clinician, feeling helps with life goals. }\end{array}$ & Promoted adherence. \\
\hline Outpatient treatment & - Participation in Assertive Community Treatment & Improved odds of filling prescriptions. \\
\hline $\begin{array}{l}\text { Medical } \\
\quad \text { medication/treatment }\end{array}$ & $\begin{array}{l}\text { - Understanding of health and consequences of } \\
\text { deciding to engage in treatment. } \\
\text { - Motivation and personal accountability for own health. }\end{array}$ & Improved medical treatment adherence. \\
\hline \multirow[t]{2}{*}{$\begin{array}{l}\text { Health promotion } \\
\text { activities/behaviors }\end{array}$} & $\begin{array}{l}\text { - Personal attitudes and beliefs about health, prior } \\
\text { experience and attitudes toward hospitalization, } \\
\text { cultural orientation, ability to negotiate health } \\
\text { system, belief treatment too } \\
\text { inconvenient/not worth effort. }\end{array}$ & $\begin{array}{l}\text { Understands the consequences of } \\
\text { engagement in health behaviors. }\end{array}$ \\
\hline & $\begin{array}{l}\text { - Adherence to psychiatric medications, sleep, daily } \\
\text { routines, use of educational media, support from } \\
\text { family/friends, past experiences with } \\
\text { mental/medical treatment improved health } \\
\text { knowledge. }\end{array}$ & $\begin{array}{l}\text { Increased participation in healthy } \\
\text { lifestyle choices. }\end{array}$ \\
\hline Treatment type & Barriers to Adherence & Findings \\
\hline \multirow{3}{*}{$\begin{array}{l}\text { Psychiatric } \\
\text { medication }\end{array}$} & - Cost burden & $50 \%$ less likely to report full adherence. \\
\hline & - Positive attitude toward positive symptoms. & $\begin{array}{l}\text { Reinforced feelings of safety when } \\
\text { non-adherent. }\end{array}$ \\
\hline & $\begin{array}{l}\text { - Substance abuse, impaired insight, high levels } \\
\text { of hostility, denial of need for treatment. }\end{array}$ & Strongly contributed to non-adherence. \\
\hline Outpatient treatment & - Missing first appointment post-hospitalization. & $\begin{array}{l}\text { Significant risk for re-hospitalization } \\
\text { during first week post-discharge. }\end{array}$ \\
\hline $\begin{array}{l}\text { Medical } \\
\text { medication/treatment }\end{array}$ & - Serious medical co-morbidities. & $\begin{array}{l}\text { Increased risk for early hospital } \\
\text { re-admission. }\end{array}$ \\
\hline \multirow[t]{2}{*}{$\begin{array}{l}\text { Health promotion } \\
\text { activities/behaviors }\end{array}$} & - Impaired intellectual capacity, cognitive deficits. & $\begin{array}{l}\text { May decline to engage in health } \\
\text { promotion activities due to lack of } \\
\text { understanding of consequences }\end{array}$ \\
\hline & $\begin{array}{l}\text { - Experiencing stigma, isolation/rejection, pain, } \\
\text { insomnia, medication side effects, emotional } \\
\text { stress, hopelessness, communication difficulties, } \\
\text { auditory hallucinations, delusions, poor dietary } \\
\text { habits, unhealthy weight, lack of transportation } \\
\text { to gym. }\end{array}$ & $\begin{array}{l}\text { Decreased likelihood of participation in } \\
\text { health promoting behaviors. }\end{array}$ \\
\hline
\end{tabular}




\section{Table 2. Summary of interventions that improve adherence}

\begin{tabular}{|c|c|c|}
\hline $\begin{array}{l}\text { Intervention } \\
\text { category }\end{array}$ & Interventions & Findings \\
\hline \multirow[t]{4}{*}{ Psychiatric medications } & $\begin{array}{l}\text { - Environmental supports: therapist } \\
\text { weekly home visits, pill counters/organizers, } \\
\text { calendars, convenient dosing, organization } \\
\text { of belongings, electronic medication } \\
\text { monitors. }\end{array}$ & $\begin{array}{l}\text { Medication adherence rates } 90-92 \% \\
\text { with supports vs. } 73 \% \text { with } \\
\text { treatment as usual. }\end{array}$ \\
\hline & $\begin{array}{l}\text { - Administration of long acting injectable } \\
\text { medications. }\end{array}$ & $\begin{array}{l}\text { Reduces all-cause hospital readmission } \\
\text { and emergency room utilization; saves } \\
\text { institutions average of } \$ 404 \text { compared } \\
\text { to use of oral medication. }\end{array}$ \\
\hline & $\begin{array}{l}\text { - Adherence Therapy and Motivational } \\
\text { interviewing: assessment of medication } \\
\text { beliefs, attitudes, knowledge and concerns, } \\
\text { education about mental illness and } \\
\text { treatment, review of benefits and drawbacks } \\
\text { of medication; strategies for relapse } \\
\text { prevention and stigma reduction. }\end{array}$ & $\begin{array}{l}\text { Significantly improved medication adherence, } \\
\text { positive and negative symptoms, } \\
\text { functioning, insight, and reduced } \\
\text { hospitalizations compared to treatment as } \\
\text { usual on one trial. }\end{array}$ \\
\hline & $\begin{array}{l}\text { - Customized Adherence Enhancement: } \\
\text { medication and psychosocial education, } \\
\text { medication routines, communication with } \\
\text { providers regarding benefits/burdens of } \\
\text { medications, adhering to medications when } \\
\text { using substances. }\end{array}$ & $\begin{array}{l}\text { Associated with good adherence to long-acting } \\
\text { injectables ( } 76 \% \text { at } 6 \text {-months) and reduction } \\
\text { in missed doses from } 46 \text { to } 10 \% \text {. }\end{array}$ \\
\hline \multirow[t]{4}{*}{ Outpatient treatment } & - Medication Management Coordinators. & $\begin{array}{l}\text { Patients attended more medication appointments } \\
\text { and at least one with psychiatrist within } \\
30 \text { days vs. usual care group. }\end{array}$ \\
\hline & - Assertive Community Treatment. & $\begin{array}{l}2.3 \% \text { greater medication possession ratios in } \\
\text { outpatient settings. }\end{array}$ \\
\hline & - Peer Support/Navigators. & $\begin{array}{l}\text { Problem-solving groups significantly reduced } \\
\text { missed medication dosages. }\end{array}$ \\
\hline & - Transitional Care. & $\begin{array}{l}\text { Significantly decreased risk for hospital } \\
\text { readmission and were } 30 \% \text { less likely to } \\
\text { be readmitted after } 1 \text { year vs. usual care. }\end{array}$ \\
\hline \multirow[t]{2}{*}{$\begin{array}{l}\text { Health promotion } \\
\text { activities }\end{array}$} & - Frequent reminders. & $\begin{array}{l}\text { Prompts from others, pill organizers, daily } \\
\text { schedules/routines (exercise, hygiene, diet, } \\
\text { spiritual growth), and use of educational media } \\
\text { facilitated participation in health promoting } \\
\text { behaviors. }\end{array}$ \\
\hline & $\begin{array}{l}\text { - Encouragement/support from family/ } \\
\text { important others. }\end{array}$ & $\begin{array}{l}\text { Enhanced likelihood of adherence to health } \\
\text { promoting behaviors. }\end{array}$ \\
\hline
\end{tabular}

